\title{
Challenges in diagnosis and treatment of cervico-cephalic arterial dissections
}

\author{
Desafios no diagnóstico e tratamento de dissecções cérvico-cefálicas
}

Adriana Bastos Conforto

\author{
Universidade de São Paulo, \\ Hospital das Clinicas da Faculdade \\ de Medicina, Divisão de Clínica \\ Neurológica, São Paulo SP, Brazil; \\ Instituto Israelita de Ensino e \\ Pesquisa Albert Einstein, São Paulo \\ SP, Brazil.

\section{Correspondence:} \\ Adriana B. Conforto; Av. Dr. Enéas de \\ Carvalho Aguiar 255/5084; 05403- \\ 000 São Paulo SP, Brasil; \\ E-mail:adriana.conforto@gmail.com \\ Conflict of interest: \\ There is no conflict of interest to \\ declare. \\ Received 20 December 2015 \\ Accepted 15 January 2016

\section{(c) BY}

I

n this number of Arquivos de Neuro-psiquiatria, Fragoso and colleagues reported a series of fourty-one cases of cervical or intracranial arterial dissections. Clinical information was retrospectively collected for dissections considered to have occurred in close temporal association with practice of physical activity, ranging from brisk walks to bungee-jumping. One patient presented an isolated Horner's syndrome while the other forty subjects presented anterior or posterior circulation infarct syndromes ${ }^{1}$. Exclusively cervical dissections were identified in twenty-nine patients while exclusively intracranial dissections were observed in eight, and combined cervical/intracranial dissections, in four cases.

Spontaneous cervical artery dissections have been associated with mechanical triggers including, but not restricted to, minor traumas that can happen in the context of physical activity. It has been hypothesized that an underlying arteriopathy leading to ultrastructural abnormalities and arterial weakness is likely present, increasing susceptibility to dissections in affected subjects ${ }^{2,3,4}$.

However, cervical dissections are often diagnosed in the absence of prior mechanical triggers. Case series cannot establish cause-effect relations between possible triggers and dissections. Still, results of an observational study suggested that mechanical triggers may indeed have a role in cervical artery dissections. In this study, Engelter et al. ${ }^{2}$ systematically evaluated the presence and type of prior cervical trauma in patients with cervical artery dissection ( $n=966)$ as well as in two other groups: age- and sex-matched patients with ischemic strokes with etiologies diferent from cervical artery dissection, $(n=651)$ and healthy subjects $(n=280)$. Prior mechanical triggers were more common in patients with cervical artery dissection than in patients with stroke due to other causes (adjusted odds ratio, 7.6 [95\%CI 5.6 - 10.2]), or in healthy subjects (adjusted odds ratio, 3.7 [95\%CI 2.4 - 5.6]). Strokes were less common and neck pain was more common in patients with dissections plus history of prior mechanical triggers than in those without. This study only included patients with cervical artery dissections. It still remains to be determined whether mechanical triggers play a role in patients with intracranial artery dissections ${ }^{4}$.

The incidence of cervical internal carotid internal artery dissection has been estimated at 2 to 3 per 100,000 per year, and of cervical vertebral artery dissection, at 1 to 1.5 per 100,000 per year ${ }^{5}$. True incidences may be underestimated because dissections are likely underdiagnosed in patients with isolated headache or neck pain ${ }^{6}$. Incidences of intracranial dissections are unknown but probably even lower ${ }^{4}$. Dissections that predominantly affect the intima and media may cause ischemic strokes, while dissections through the adventitia may lead to formation of pseudoaneurysms or even to subarachnoid hemorrhage, when they spread intracranially. Ischemic strokes can occur due to arterial occlusion by a mural hematoma, or by embolism from thrombi that form on the dissected artery ${ }^{3}$. Despite these relative low incidences, this condition is responsible for up to $10-25 \%$ of ischemic strokes in young patients. Therefore, dissection should be kept in mind in the differential diagnosis of stroke etiology, whether or not patients report histories of mechanical triggers.

Therapeutic implications of a diagnosis of dissection are still a matter of debate. The Cervical Artery Dissection in Stroke Study (CADISS) ${ }^{7}$ was a randomized controlled clinical trial that compared anticoagulation and antiplatelet treatment in two hundred and fifty patients with cervical carotid or vertebral artery dissections with onset of symptoms within seven days. The choice of antiplatelet drug or anticoagulant drug was at the discretion of the local physician. Antiplatelet 
treatments included aspirin, dipyridamole, or clopidogrel alone or in combination. Twenty-eight percent of the patients received a combination of aspirin and clopidogrel.

This study was planned as a phase 2 feasibility trial to calculate the rate of recurrent stroke and the sample size required for a phase 3 trial. The primary endpoint was ipsilateral stroke or death within 3 months of randomization in the intent-to-treat population.

In CADISS, ${ }^{8}$ twenty-six of the patients did not have strokes or transient ischemic attacks, and central review of imaging failed to confirm dissection in fifty-two patients. Stroke recurrence rates were low and not statistically significantly different between the two groups ( $2 \%$ in the antiplatelet arm and $1 \%$ in the anticoagulant arm in the intent-to-treat population). The results highlighted the difficulties in radiological confirmation of dissection as well in stratification of patients at greater risk of stroke.
The low risk of recurrent stroke in the studied patients contributed to the prediction that a trial would need to include at least 4876 patients in each group, in order to detect a $1 \%$ difference in stroke or death between the two treatments in patients with similar eligibility criteria. The study was hence not powered to provide final answers about the comparison between antiplatelet and anticoagulant drugs, particularly in patients diagnosed very early after stroke. The best strategy in these cases is still unclear. Whether dual antiplatelet therapy is more likely to prevent recurrent strokes than single antiplatelet therapy also remains to be determined. In addition, there are no evidence-based treatments for intracranial dissections. While the results of CADISS do not encourage widespread use of anticoagulants in all cases of cervical arterial dissections, there are clinically relevant questions that remain unanswered.

\section{References}

1. Fragoso YD, Adoni T, Amaral LLF, Braga FT, Brooks JBB, Campos CS et al. Cerebrum-cervical arterial dissection in adults during sports and recreation. Arquivos Neuropsiquiatr. 2016;74(4):275-79. doi:10.1590/0004-282X20150150

2. Engelter ST, Grond-Ginsbach C, Metso TM, Metso AJ, Kloss M, Debette $S$ et al. Cervical artery dissection: trauma and other potential mechanical trigger events. Neurology. 2013;80(21):1950-7. doi:10.1212/WNL.0b013e318293e2eb

3. Campos-Herrera CR, Scaff M, Yamamoto FI, Conforto AB. Spontaneous cervical artery dissection: an update on clinical and diagnostic aspects. Arq Neuropsiquiatr. 2008;66(4):922-7. doi:10.1590/S0004-282X2008000600036

4. Debette S, Compter A, Labeyrie M, Uyttenboogaart M, Metso TM, Majersik JJ et al. Epidemiology, pathophysiology, diagnosis, and management of intracranial artery dissection. Lancet Neurol 2015;14(6):640-54. doi:10.1016/S1474-4422(15)00009-5

\footnotetext{
5. Thomas LC, Rivett DA, Attia JR, Levi CR. Risk factors and clinical presentation of craniocervical arterial dissection: a prospective study. BMC Musculoskeletal Disorders 2012;13:164. doi:10.1186/1471-2474-13-164

6. Blum CA, Yaghi S. Cervical artery dissection: a review of the epidemiology, pathophysiology, treatment and outcome. Arch Neurosci. 2015;2(4):e26670. doi: 10.5812/archneurosci.26670

7. Markus HS, Hayter E, Levi C, Feldman A. Antiplatelet treatment compared with anticoagulation treatment for cervical artery dissection (CADISS): a randomised trial. Lancet Neurol. 2015;14(4):361-7. doi:10.1016/S1474-4422(15)70018-9

8. Kasner SE. CADISS: a feasibility trial that answered its question. Lancet Neurol. 2015;14(4):342-3. doi:10.1016/S1474-4422(14)70271-6
} 